\title{
Perfil celular do escarro induzido e sangue periférico na doença pulmonar obstrutiva crônica*
}

\author{
Induced sputum and peripheral blood cell profile \\ in chronic obstructive pulmonary disease
}

\author{
Rogerio Rufino', Cláudia Henrique da Costa', Heitor Siffert Pereira de Souza ${ }^{2}$, \\ Kalil Madi ${ }^{3}$, José Roberto Lapa e Silva ${ }^{4}$
}

\begin{abstract}
Resumo
Objetivo: Determinar o perfil celular e subgrupos linfocitários $\mathrm{CD}^{+}$e $\mathrm{CD} 8^{+}$no escarro induzido (El) e sangue venoso periférico (SVP) de pacientes com doença pulmonar obstrutiva crônica (DPOC). Métodos: Foram quantificadas as celularidades total e específica, incluindo subgrupos linfocitários T CD4+ e CD8 ${ }^{+}$, do El e SVP de 85 pessoas (38 pacientes com DPOC sem agudização, 29 tabagistas sem obstrução e 18 não-tabagistas). Os testes não-paramétricos de Mann-Whitney e Spearman foram usados na análise estatística, considerando como significante o $p<0,05$. Resultados: Os neutrófilos, eosinófilos e linfócitos T CD8 ${ }^{+}$do El estavam aumentados $(p=0,005, p<0,05$ e $p<0,05)$ e o percentual de macrófagos encontrava-se reduzido $(\mathrm{p}=0,003)$ nos pacientes com DPOC, em relação aos não-tabagistas. A correlação linear de cada tipo celular do El com o volume expiratório forçado no primeiro segundo (VEF) , a capacidade vital forçada (CVF), e VEF $/$ CVF foi fraca $\left(r^{2}<0,1\right)$. Os eosinófilos e os linfócitos T CD8 ${ }^{+}$também estavam aumentados no SVP ( $p=0,04$ e $\left.p=0,02\right)$. Conclusões: Em pacientes com DPOC estável, as células T CD8 ${ }^{+}$estavam aumentadas no SVP, embora a leucometria total tenha se mantido em valores semelhantes aos dos outros dois grupos estudados, indicando possível envolvimento inflamatório sistêmico. A contagem dos linfócitos T CD8 ${ }^{+}$no sangue pode ser útil como marcador de inflamação sistêmica e auxiliar na identificação de tabagistas que já possuem padrão inflamatório de DPOC.
\end{abstract}

Descritores: Escarro; Linfócitos T; Neutrófilos; Macrófagos; Eosinófilos; Doença pulmonar obstrutiva crônica.

\begin{abstract}
Objective: To determine cell profiles, as well as to identify CD4 ${ }^{+}$and CD8 ${ }^{+}$lymphocyte subgroups, in induced sputum (IS) and peripheral venous blood (PVB) of patients with chronic obstructive pulmonary disease (COPD). Methods: Total cell counts and counts of individual cell types, including $\mathrm{CD}^{+}$and $\mathrm{CD} 8^{+}$T lymphocytes, were determined in the $1 \mathrm{~S}$ and PVB of 85 subjects (38 with COPD without exacerbation, 29 smokers without obstruction and 18 nonsmokers). Mann-Whitney and Spearman non-parametric tests were used in the statistical analysis, and values of $p<0.05$ were considered statistically significant. Results: Comparing the 1S of subjects with COPD to that of nonsmokers, neutrophil, eosinophil and CD8 ${ }^{+} \mathrm{T}$ lymphocyte counts were higher (respectively $p=0.005, p<0.05$ and $p<0.05$ ), whereas the percentage of macrophages was lower $(p=0.003)$. There were weak linear correlations $\left(r^{2}<0.1\right)$ between each cell type in 15 and forced expiratory volume in one second (FEV $)$, forced vital capacity (FVC) and $\mathrm{FEV}_{1} / \mathrm{FVC}$ ratio. Eosinophil and CD8 ${ }^{+} \mathrm{T}$ lymphocyte counts were also higher in PVB ( $p=0.04$ and $p=0.02$ ). Conclusions: In patients with stable COPD, CD8 ${ }^{+}$T lymphocyte counts were higher in PVB, whereas total leukocyte counts were similar to those of the other two groups analyzed, suggesting systemic inflammatory involvement. The CD8 ${ }^{+} \mathrm{T}$ lymphocyte count in blood can be a useful marker of systemic inflammation and can help identify smokers who already present a COPD inflammatory pattern.
\end{abstract}

Keywords: Sputum; T-lymphocytes; Neutrophils; Macrophages; Eosinophils; Pulmonary disease, chronic obstructive.

\footnotetext{
* Trabalho realizado na Universidade do Estado do Rio de Janeiro e no Laboratório Multidisciplinar de Pesquisa da Universidade Federal do Rio de Janeiro - UFRJ Rio de Janeiro (RJ) Brasil.

1. Professor Adjunto de Pneumologia e Tisiologia da Faculdade de Ciências Médicas da Universidade do Estado do Rio de Janeiro - UERJ - Rio de Janeiro (RJ) Brasil.

2. Professor Adjunto de Clínica Médica da Faculdade de Medicina. Universidade Federal do Rio de Janeiro - UFRJ - Rio de Janeiro (RJ) Brasil.

3. Professor Titular do Departamento de Patologia da Faculdade de Medicina. Universidade Federal do Rio de Janeiro - UFRJ - Rio de Janeiro (RJ) Brasil.

4. Professor Titular de Pneumologia e Tisiologia da Faculdade de Medicina. Universidade Federal do Rio de Janeiro - UFRJ - Rio de Janeiro (RJ) Brasil.

Endereço para correspondência: José Roberto Lapa e Silva. Laboratório Multidisciplinar, Hospital Universitário Clementino Fraga Filho,

Universidade Federal do Rio de Janeiro, Av. Brigadeiro Tromposki, s/n, llha do Fundão, CEP 21941-590, Rio de Janeiro, RJ, Brasil.

Tel 5521 2562-2669. Fax 5521 2290-3520. E-mail: jrlapa.ntg@terra.com.br

Recebido para publicação em 27/9/2006. Aprovado, após revisão, em 25/2/2007.
} 


\section{Introdução}

A doença pulmonar obstrutiva crônica (DPOC) é caracterizada, desde 1998, como uma doença inflamatória das vias respiratórias, do parênquima e dos vasos pulmonares, evoluindo com lenta, progressiva e irreversivel obstrução ao fluxo expiratório. ${ }^{(1)}$ A Organização Mundial de Saúde prevê que, nos próximos 15 anos, ela se tornará a $5^{\text {a }}$ doença em prevalência no mundo e a $3^{\text {a }}$ causa de mortalidade. ${ }^{(1)}$

Em algumas circunstâncias, como a infecção e o fumo, os linfócitos e os monócitos migram em grande quantidade para o pulmão.(2) Lá, eles são ativados e geram uma reação inflamatória que, ao longo dos anos, acarreta alterações na estrutura e função pulmonares. Este processo inflamatório acontece nas pequenas vias respiratórias (menores que $2 \mathrm{~mm}$ ), sendo normalmente tênue e ininterrupto. Postula-se que a responsável pela ruptura das estruturas epiteliais de sustentação alveolar seja a inflamação persistente das vias respiratórias distais em pacientes com DPOC. Assim, os alvéolos e ductos alveolares coalescem de forma irregular e definitiva. $^{(3)}$

As células inflamatórias recrutadas na DPOC liberam elastases, colagenases e produtos oxidantes que, superpostos aos oxidantes inalados da fumaça do cigarro, atuam modificando os componentes da matriz extracelular. ${ }^{(4)}$ Assim, o pulmão adquire um novo modelo, deformado e irreversível, com estiramento e desaparecimento de septos alveolares, formando espaços aéreos maiores, associados às áreas de compressões brônquicas, e desenvolvendo regiões heterogêneas de hiperinsuflação. ${ }^{(4,5)}$

$\mathrm{Na}$ década de 90, grupos de estudo da patogênese da DPOC descreveram o desequilíbrio de substâncias liberadas pelos neutrófilos e macrófagos pulmonares, propondo o conceito de um processo único de desenvolvimento fisiopatológico da doença. Deste modo, as teorias envolvendo elastase/ antielastase e oxidante/antioxidante começaram a ser interpretadas conjuntamente. Estas informações foram reunidas, constituindo uma proposta de modelo inflamatório da DPOC, contendo, predominantemente, informações da participação de macrófagos e neutrófilos. ${ }^{(6-8)}$

Nesta mesma década, outros estudos com amostras de biópsia pulmonar demonstraram que os linfócitos T CD8 ${ }^{+}$estavam significativamente aumentados nas vias respiratórias inferiores dos pacientes com DPOC. Assim, o modelo inflamatório, até então composto por neutrófilos e macrófagos, apresentava novo ator celular, o linfócito $\mathrm{T} \mathrm{CD} 8^{+} .(9,10)$

Em 1995, alguns autores ${ }^{(11)}$ postularam que as pessoas que desenvolveriam a DPOC seriam aquelas que já apresentavam diminuição fenotípica de células $\mathrm{CD} 4^{+}$ou aumento fenotípico das células $\mathrm{CD} 8^{+}$ no sangue. Desta forma, as alterações linfocitárias do sangue poderiam estar envolvidas no mecanismo fisiopatogênico da DPOC, fato que ainda não está confirmado pela literatura. ${ }^{(6)}$

Mais recentemente, alguns autores demonstraram a presença de linfócitos $\mathrm{T}$ e macrófagos na parede das vias aéreas de tabagistas, enquanto que os neutrófilos são preferencialmente encontrados na luz brônquica. ${ }^{(7,10)}$ Estes estudos observaram que mesmo os tabagistas que não apresentam sintomas respiratórios desenvolvem resposta inflamatória pulmonar crônica resultante da agressão causada pelo tabagismo. No entanto, somente uma pequena parcela dos tabagistas desenvolve a DPOC, sugerindo que o processo inflamatório possa evoluir de forma diferente entre estes pacientes. 0 estudo do perfil celular dos dois grupos é fundamental para que haja uma melhor compreensão da patogênese da doença.

0 escarro induzido (El), inicialmente utilizado para o diagnóstico de câncer de pulmão e, posteriormente, para doenças infecciosas, foi metodologicamente re-estudado e aceito como um instrumento de investigação da patogenia da asma. $^{(12)} \mathrm{Na}$ DPOC, o El ainda está sendo agregado como uma ferramenta para o aprimoramento do conhecimento do processo inflamatório, devido à dificuldade de reprodutibilidade dos achados celulares e bioquímicos provenientes deste método. ${ }^{(13)}$

Este estudo tem como objetivo analisar o perfil celular do El e do sangue periférico de pacientes com DPOC, tabagistas sem obstrução brônquica e controles não tabagistas, e tentar estabelecer o perfil da celularidade evidenciada nos pacientes com doença obstrutiva.

\section{Métodos}

0 projeto foi aprovado pelo Comitê de Ética em Pesquisa do Hospital Universitário Clementino Fraga Filho, da Universidade Federal do Rio de Janeiro, e todos os indivíduos do estudo assinaram Termo de Consentimento Livre e Esclarecido. Foram avaliadas 
85 pessoas, sendo 38 pacientes com DPOC, 29 tabagistas e 18 não-tabagistas sem obstrução ao fluxo aéreo. 0 diagnóstico de DPOC foi feito com base nos critérios do Global Initiative for Chronic Obstructive Lung Disease (GOLD), ${ }^{(1)}$ sendo 3 definidos como leves, 18 como moderados e 17 como graves.

Para participar do estudo, os indivíduos deveriam preencher todos os critérios a seguir: a) apresentar valor de $\alpha-1$ antitripsina dentro da normalidade; b) não ter tido nenhuma infecção respiratória por, no mínimo, 4 semanas; c) ter teste de função respiratória com prova broncodilatadora negativa há, no máximo, 1 semana; ${ }^{(14)}$ e d) não ter história de atopia cutânea ou de vias respiratórias superiores e/ou inferiores.

Os seguintes critérios de exclusão foram considerados: a) utilização de corticóide inalável ou sistêmico há menos de 1 mês; b) utilização de antibiótico há menos de 1 mês; c) história pregressa de tuberculose; e d) infecção com vírus da imunodeficiência adquirida humana (HIV).
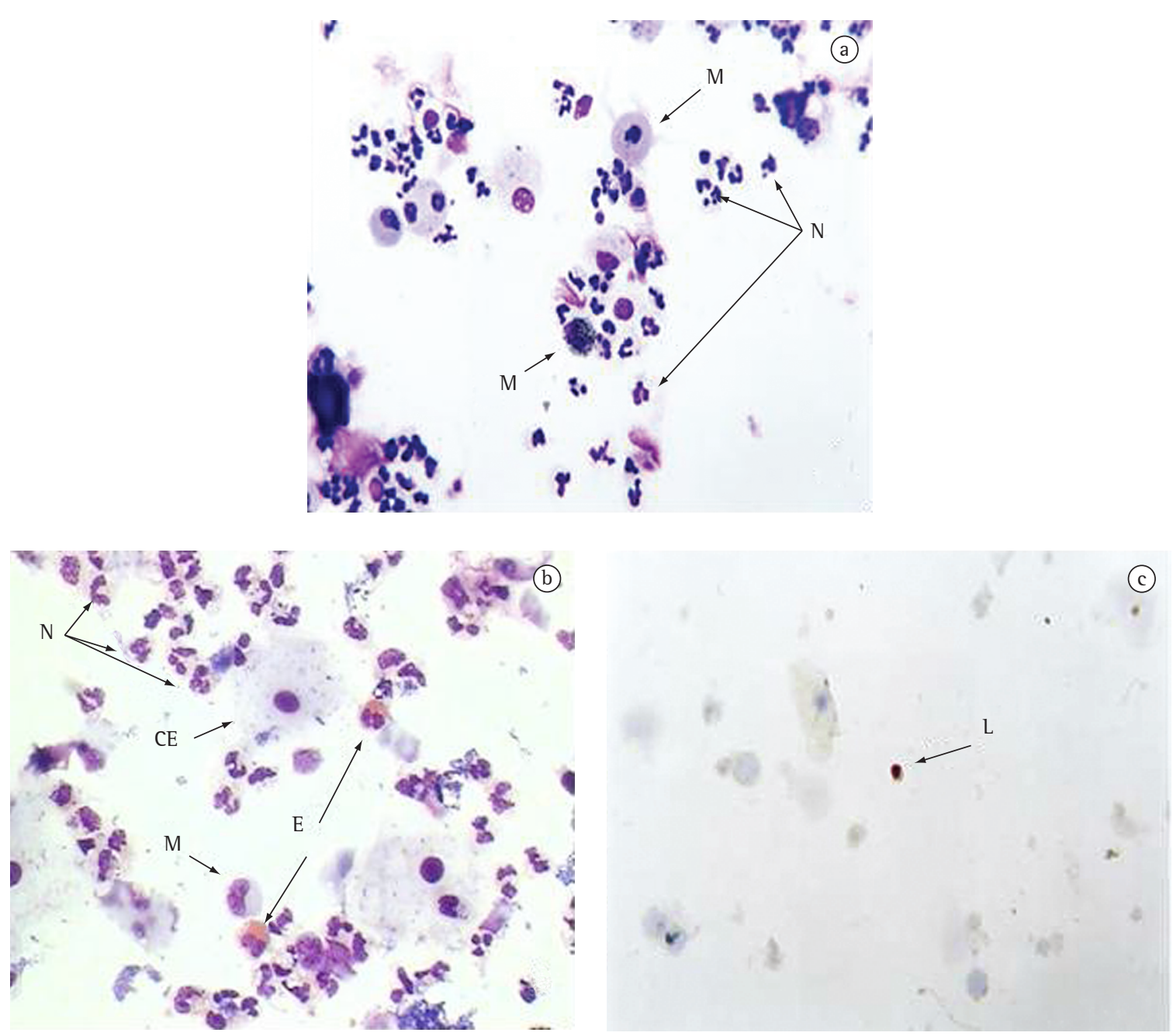

Figura 1 - Painel dos achados celulares do escarro de três pacientes com doença pulmonar obstrutiva crônica pelo método do escarro induzido. a) Macrófagos com pigmentos pretos (antracóticos) distribuídos no interior do citoplasma (seta menor) e grandes pseudópodos (seta longa). 0 padrão do escarro induzido é bicelular, neutrofílico-macrofílico (Coloração de Hematoxilina-Eosina. Médio-aumento); b) 0 padrão do escarro induzido é multicelular, neutrofílicomacrofílico-eosinófilico, com identificação de células escamosas da orofaringe (Coloração de Hematoxilina-Eosina. Médio-aumento); e c) Marcação imunoperoxidase para células CD8 ${ }^{+}$de somente um linfócito (Médio-aumento). CE: células escamosas; E: eosinófilos; L: linfócitos; M: macrófagos; e N: neutrófilos. 
Três grupos foram constituídos:

1) DPOC: pacientes tabagistas (fumantes ativos) com carga tabágica acima de 20 anos-maço, sem história de atopia, $\mathrm{VEF}_{1} / \mathrm{CVF} \leq 70 \%$, e prova broncodilatadora negativa, de acordo com os critérios da American Thoracic Society (ATS);(14)

2) Tabagista: pacientes tabagistas (fumantes ativos) com carga tabágica acima de 20 anos-maço, sem história de atopia, $\mathrm{VEF}_{1} / \mathrm{CVF}>70 \%, \mathrm{VEF}_{1}>80 \%$ e prova broncodilatadora negativa, de acordo com os critérios da ATS; ${ }^{(14)} \mathrm{e}$

3) Não-tabagista:indivíduossem história de tabagismo e de atopia, $\mathrm{VEF}_{1} / \mathrm{CVF}>70 \%, \mathrm{VEF}_{1}>80 \%$ e prova broncodilatadora negativa, de acordo com os critérios da ATS. ${ }^{(14)}$

A coleta do sangue venoso foi realizada imediatamente antes da coleta do escarro. A indução do escarro foi feita seguindo a técnica de Pin e colaboradores ${ }^{(12)}$ com modificações, após pré-tratamento com $400 \mu \mathrm{g}$ de salbutamol inalado, para prevenir sintomas de obstrução brônquica. A modificação do método consistiu em utilizar concentrações crescentes de salina hipertônica a 2, 3 e 4\% (10 mL por solução). Cada etapa da nebulização teve a duração de $7 \mathrm{~min}$, totalizando $21 \mathrm{~min}$. 0 aparelho utilizado para a nebulização ultra-sônica foi o Ultra-NEB'99 (DeVilbiss, Somerset, PA, EUA). 0 material coletado foi imediatamente encaminhado sob gelo para análise laboratorial.

Foram selecionados os grumos e a parte mais espessa do escarro e centrifugados a $1.500 \mathrm{rpm}$, na temperatura de $4{ }^{\circ} \mathrm{C}$, por 20 min. 0 sobrenadante foi armazenado em Eppendorf estéril para dosagens da fase sol. Em seguida, o restante foi tratado com ditiotreitol (Sigma Chemical, St. Louis, MO, EUA), posto imerso em 'banho Maria', na temperatura de $37^{\circ} \mathrm{C}$, por $10 \mathrm{~min}$, e centrifugado a $1.500 \mathrm{rpm}$, na temperatura de $4{ }^{\circ} \mathrm{C}$, durante $10 \mathrm{~min}$. Nesta fase, coletava-se uma nova amostra de sobrenadante, que foi armazenada em tubo de Eppendorf. 0 precipitado foi re-suspenso com $1000 \mu$ lde solução estéril salina tamponada com fosfato. Separava-se uma alíquota para estudo da viabilidade celular por meio da coloração por azul de Tripan, usando a câmara de Neubauer para contagem. Os citocentrífugos foram preparados com 25.000 células por poço (cytospin), fixadas em clorofórmio:acetona. Uma lâmina corada com Diff-Quick foi utilizada para contagem diferencial celular, usando-se o microscópio Nikon 155196, na ocular 10x e na objetiva 40x e com aumento de 400x (Figura 1).

0 material coletado que apresentava o percentual de células escamosas pela coloração de hematoxilina-eosina superior a 70\% do total de células encontradas, o número de células não-escamosas inferior a 200, ou a viabilidade celular inferior a $50 \%$, era descartado. ${ }^{(12)}$

Foram realizadas marcações das subpopulações linfocitárias das células $\mathrm{T} \mathrm{CD}^{+}$e $\mathrm{CD}^{+}$, com os respectivos anticorpos humanos monoclonais CD4 (clone QA4120, código C-1805, lote 116H4875; Sigma Chemical) e CD8 (clone UCHT-4, código C-7423, lote 40K4830, Sigma Chemical). A técnica imuno-histoquímica utilizada foi a de complexo avidina-biotina-peroxidase (Figura 1).

A contagem das células no sangue periférico foi feita por meio de citometria de fluxo pelo FACScount (BD Biosciences Imunocytometry

Tabela 1 - Dados demográficos e valores da espirometria.

\begin{tabular}{|c|c|c|c|c|c|c|c|c|}
\hline \multirow[t]{2}{*}{ Grupos } & \multirow[t]{2}{*}{$\begin{array}{c}\text { Gênero } \\
\mathrm{M} / \mathrm{F}\end{array}$} & \multirow[t]{2}{*}{$\begin{array}{l}\text { ldade } \\
\text { (anos) }\end{array}$} & \multirow[t]{2}{*}{$\begin{array}{c}\text { CT } \\
\text { (anos-maço) }\end{array}$} & \multirow[t]{2}{*}{$\begin{array}{c}\mathrm{VEF}_{1} / \mathrm{CVF} \\
(\%)\end{array}$} & \multicolumn{2}{|c|}{$\mathrm{VEF}_{1}$} & \multicolumn{2}{|c|}{ CVF } \\
\hline & & & & & \multicolumn{2}{|c|}{$(\mathrm{L} \%)$} & \multicolumn{2}{|c|}{ (L \%) } \\
\hline Não-tabagista & $9 / 9$ & $\begin{array}{c}41,37 \\
(12,11)\end{array}$ & - & $\begin{array}{c}80,72 \\
(7,26)\end{array}$ & $\begin{array}{c}2,99 \\
(0,84)\end{array}$ & $\begin{array}{l}103,1 \\
(15,96)\end{array}$ & $\begin{array}{c}3,69 \\
(0,94)\end{array}$ & $\begin{array}{l}105,5 \\
(14,46)\end{array}$ \\
\hline Tabagistas & $16 / 13$ & $\begin{array}{r}53,79^{c} \\
(13,04)\end{array}$ & $\begin{array}{c}30,04 \\
(14,13)\end{array}$ & $\begin{array}{c}76,97 \\
(5,23)\end{array}$ & $\begin{array}{c}2,66 \\
(0,78)\end{array}$ & $\begin{array}{c}94,48 \\
(17,07)\end{array}$ & $\begin{array}{c}3,48 \\
(0,92)\end{array}$ & $\begin{array}{l}100,8 \\
(17,30)\end{array}$ \\
\hline DPOC & $26 / 12$ & $\begin{array}{r}63,45^{b} \\
(10,28) \\
\end{array}$ & $\begin{array}{r}37,97^{a} \\
(14,34) \\
\end{array}$ & $\begin{array}{c}54,55^{\mathrm{b}, \mathrm{d}} \\
(9,07)\end{array}$ & $\begin{array}{c}1,45^{\mathrm{b}, \mathrm{d}} \\
(0,68)\end{array}$ & $\begin{array}{c}59,00^{\mathrm{b}, \mathrm{d}} \\
(24,80)\end{array}$ & $\begin{array}{c}2,59^{\mathrm{b}, \mathrm{d}} \\
(0,97) \\
\end{array}$ & $\begin{array}{c}85,05^{\mathrm{b}, \mathrm{d}} \\
(27,29)\end{array}$ \\
\hline
\end{tabular}

Os valores estão expressos em média e desvio padrão; M: masculino; F: feminino; CT: carga-tabágica; VEF, volume expiratório forçado no primeiro segundo; CVF: capacidade vital forçada; DPOC: doença pulmonar obstrutiva crônica; ${ }^{a} p<0,05$ para a diferença entre DPOC e tabagista; ${ }^{b} p<0,01$ para a diferença entre DPOC e tabagista; ${ }^{c} p<0,05$ para a diferença entre tabagistas e não-tabagistas; $\mathrm{e}^{\mathrm{d}} \mathrm{p}<0,01$ para a diferença entre DPOC e não-tabagistas. 
Systems, San Jose, CA, EUA), a qual fornecia o número absoluto e percentual dos leucócitos e seus subtipos e das subpopulações dos linfócitos T CD4 ${ }^{+}$ e $\mathrm{CD}^{+}$. Os resultados foram expressos em mediana e faixa ou média e desvio-padrão. Inicialmente, a distribuição das amostras foi analisada utilizando os testes Kolmogorov-Smirnov; o teste não-paramétrico Mann-Whitney duplo caudal foi usado para a análise entre os grupos; e o de Spearman, para as correlações lineares; foi considerada significância estatística quando $p$ fosse inferior a 0,05.

\section{Resultados}

Os dados demográficos de todos os indivíduos do estudo e as médias das análises funcionais são fornecidos no Tabela 1.

A análise da contagem celular feita no El demonstrou redução significativa do percentual de células epiteliais não-escamosas no grupo de pacientes com DPOC, quando comparado ao dos não-tabagistas, respectivamente, $0,37 \pm 0,78 \% \mathrm{e}$ $1,06 \pm 1,51 \%, p=0,049$ (Tabela 2).

0 valor percentual de macrófagos estava significativamente reduzido nos pacientes com DPOC $(37,68 \pm 22,47 \%)$, quando comparado aos tabagistas $(55,86 \pm 21,84 \%, p=0,002)$ e aos não-tabagistas $(58,67 \pm 23,90 \%, p=0,005)$ (Tabela 2 e Figura 2). Da mesma forma, o valor absoluto de macrófagos também estava reduzido nos pacientes com DPOC $(155,92 \pm 133,75)$ em relação aos controles nãotabagistas $(185,47 \pm 125,33, p<0,01)$ (Tabela 2).

Ao contrário dos macrófagos, o percentual de neutrófilos estava significativamente elevado no material recolhido dos pacientes com DPOC $(58,05 \pm 25,28 \%)$, em relação ao encontrado no escarro dos tabagistas $(40,17 \pm 22,50 \%, p=0,019)$ e dos não-tabagistas $(39,33 \pm 24,34 \%, p=0,003)$ (Tabela 2 e Figura 2).

Quando correlacionado o percentual de neutrófilos recolhidos no El com o $\mathrm{VEF}_{1}$, a CVF e a relação $V_{E F_{1}} / C V F$, verificava-se a existência de uma corre-

Tabela 2 - Valores absolutos da celularidade no escarro induzido e no sangue periférico.

\begin{tabular}{|c|c|c|c|}
\hline & \multicolumn{3}{|c|}{ Escarro induzido } \\
\hline & Não tabagista & Tabagista & DPOC \\
\hline Total de células $\left(\times 10^{5} / \mathrm{mL}\right)$ & $586,95(268,29)$ & $603,90(232,94)$ & $637,13(392,68)$ \\
\hline Macrófagos (× 105/mL) & $185,47(125,33)$ & $208,43(111,19)^{c}$ & $155,92(133,75)^{\mathrm{e}}$ \\
\hline Macrófagos (\%) & $58,67(23,90)$ & $55,86(21,84)$ & $37,68(22,40)^{b, e}$ \\
\hline Eosinófilos $\left(\times 10^{5} / \mathrm{mL}\right)$ & $2,47(5,10)$ & $7,37(17,05)$ & $20,66(44,08)^{d}$ \\
\hline Eosinófilos (\%) & $0,22(0,55)$ & $0,38(0,94)$ & $1,03(2,66)$ \\
\hline Neutrófilos (× 105/mL) & $141,00(112,39)$ & $199,40(159,12)^{c}$ & $312,37(396,30)^{\mathrm{e}}$ \\
\hline Neutrófilos (\%) & $39,33(24,34)$ & $40,17(22,50)$ & $58,05(25,28)^{a, e}$ \\
\hline Células epiteliais $\left(\times 10^{5} / \mathrm{mL}\right)$ & $2,50(3,79)$ & $3,90(8,90)$ & $1,50(2,29)^{\mathrm{e}}$ \\
\hline Linfócitos (× 105/mL) & $0,89(1,53)$ & $1,37(2,68)$ & $2,58(5,37)$ \\
\hline Linfócitos (\%) & $0,22(0,55)$ & $0,38(0,94)$ & $1,03(2,66)$ \\
\hline $\mathrm{CD}^{+}\left(\times 10^{5} / \mathrm{mL}\right)$ & $0,63(1,01)$ & $0,47(0,90)$ & $0,71(2,05)$ \\
\hline $\mathrm{CD}^{+}\left(\times 10^{5} / \mathrm{mL}\right)$ & $0,26(0,45)$ & $1,00(2,24)$ & $1,89(3,54)^{\mathrm{e}}$ \\
\hline \multirow[t]{3}{*}{ CD4/CD8 } & $0,42(1,02)$ & $1,89(3,54)$ & $0,10(0,25)$ \\
\hline & \multicolumn{3}{|c|}{ Sangue periférico $\left(\mathrm{mm}^{3}\right)$} \\
\hline & Não tabagista & Tabagista & DPOC \\
\hline Total de células & $6593,68(1939,08)$ & $7011,07(1381,13)$ & $6701,66(1290,57)$ \\
\hline Neutrófilos & $4167,95(1752,96)$ & $4559,75(1368,14)$ & $4386,57(1188,30)$ \\
\hline Eosinófilos & $186,53(114,31)$ & $233,89(181,19)$ & $306,58(189,40)^{\mathrm{e}}$ \\
\hline Linfócitos & $1857,95(705,37)$ & $1768,21(564,54)$ & $1611,58(576,95)$ \\
\hline $\mathrm{CD}^{+}$ & $583,42(450,13)$ & $985,14(262,11)$ & $908,53(347,64)$ \\
\hline $\mathrm{CD}^{+}$ & $471,16(137,57)$ & $536,07(204,70)$ & $502,26(175,11)^{e}$ \\
\hline CD4/CD8 & $2,22(1,01)$ & $2,02(0,70)$ & $1,99(0,87)^{\mathrm{e}}$ \\
\hline
\end{tabular}

Os valores estão expressos em média e desvio-padrão; DPOC: doença pulmonar obstrutiva crônica; ${ }^{a} p<0,05$ para a diferença entre DPOC e tabagistas; ${ }^{b} p<0,01$ para a diferença entre DPOC e tabagistas; ${ }^{c} p<0,05$ para a diferença entre tabagistas e não-tabagistas; ${ }^{d} p<0,05$ para a diferença entre DPOC e não-tabagistas; e ${ }^{e} p<0,01$ para a diferença entre DPOC e não-tabagistas. 
lação linear fraca entre esses parâmetros, com os valores de $r^{2}$ respectivamente de 0,108, 0,078 e 0,093, $p<0,05$. 1sto também se repetiu com a correlação linear de macrófagos, eosinófilos, células epitelias, linfócitos e as suas subpopulações no El com os mesmos parâmetros espirométricos $\left(\mathrm{r}^{2}<0,1\right)$.

Houve aumento do valor absoluto de eosinófilos nos pacientes com DPOC em comparação ao dos controles não-tabagistas $(p<0,05)$. No entanto, esta diferença não foi verificada ao se comparar os tabagistas com os pacientes com DPOC (Tabela 2).

Embora tenha sido verificada uma tendência de aumento do percentual de linfócitos no El dos pacientes com DPOC $(1,03 \pm 2,66 \%)$ em relação ao dos tabagistas $(0,38 \pm 0,94 \%)$ e não-tabagistas $(0,22 \pm 0,55 \%)$, essa diferença não foi estatisticamente significativa. Quando analisados os valores absolutos das subpopulações linfocitárias $\mathrm{CD}^{+}$ e $\mathrm{CD}^{+}$, observou-se tendência de aumento da primeira linhagem e de aumento significativo da última ( $<<0,01)$, no grupo de pacientes com DPOC, em relação ao dos não-tabagistas, fato este que contribuiu para a diminuição da relação CD4/CD8 no grupo de DPOC.

0 grupo de tabagistas apresentava tendência à diminuição das células $\mathrm{CD}^{+}$e ao aumento das

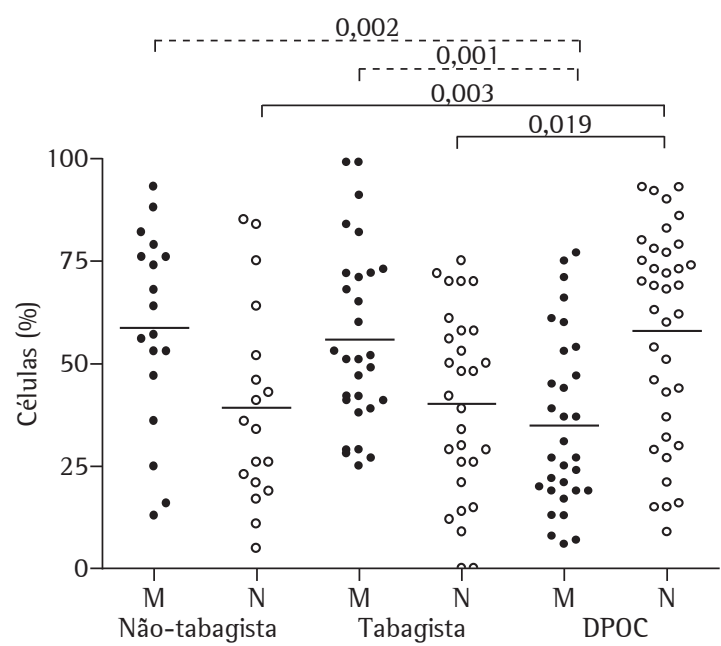

Figura 2 - Valores percentuais de macrófagos e neutrófilos observados no escarro induzido. A linha horizontal entre os pontos vazios ou cheios representa a média dos valores. A análise estatística foi realizada por meio do teste de Mann-Whitney, duplo caudal. M: macrófagos; $\mathrm{N}$ : neutrófilos; e DPOC: doença pulmonar obstrutiva crônica. células $\mathrm{T}$ CD8 ${ }^{+}$, sem significância estatística em relação ao grupo de não-tabagistas (Tabela 2).

No que tange à quantificação das células no sangue, foi verificado que as populações de neutrófilos e linfócitos totais foram semelhantes nos três grupos de estudo. No entanto, quando analisadas as subpopulações linfocitárias, observou-se aumento significativo das células $\mathrm{T} \mathrm{CD}^{+}$no sangue periférico dos pacientes com DPOC $(32,29 \pm 9,55 \%)$, quando comparadas às dos controles não-tabagistas $(27,72 \pm 10,7 \%, p=0,036)$, mas não com relação às dos tabagistas (30,76 $\pm 7,75 \%)$. Apesar da ausência de significância estatística, as células T CD4 ${ }^{+}$apresentaram tendência de aumento nos grupos de tabagistas e pacientes com DPOC em relação aos controles não-tabagistas. (Tabela 2 e Figura 3)

Quanto aos eosinófilos, houve aumento do percentual destas células no sangue de pacientes com DPOC $(4,5 \pm 2,6 \%)$ com relação ao dos não tabagistas $(2,83 \pm 1,5 \%, p=0,021)$ (Tabela 2).

Assim como no escarro, os neutrófilos, os linfócitos e as suas subpopulações no sangue venoso também demonstraram fraca correlação com os parâmetros espirométricos $\left(\mathrm{r}^{2}<0,1\right)$.

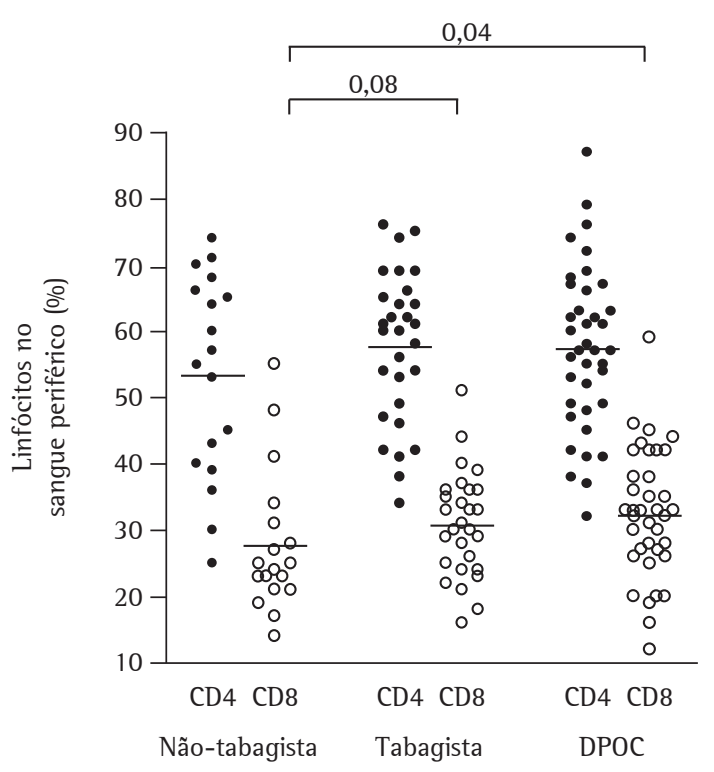

Figura 3 - Valores percentuais dos subtipos linfocitários no sangue periférico - linfócitos $\mathrm{T} \mathrm{CD}^{+}(\bullet)$ e linfócitos $\mathrm{T} \mathrm{CD}^{+}(\mathrm{O})$ - junto com os valores médios $(-)$. A análise estatística foi realizada com o teste de Mann-Whitney, duplo caudal. Verifica-se aumento significativo de linfócitos $\mathrm{T} \mathrm{CD}^{+}$nos pacientes com doença pulmonar obstrutiva crônica (DPOC) em relação aos controles não-tabagistas. 


\section{Discussão}

A DPOC tem como característica a inflamação crônica, com expressão fenotípica das células imunitárias T $\mathrm{CD}^{+}$, o que já a diferencia de muitas doenças inflamatórias não-infecciosas no pulmão. Além disto, há um desequilíbrio de substâncias proteolíticas/antiproteolíticas e oxidantes/antioxidantes, que preferencialmente acontece nas áreas mais distais do pulmão. Este conjunto de variáreis dificulta dimensionar como e onde atuar para evitar a progressão da doença. ${ }^{(1,6,15)}$

Alguns autores propõem que a inflamação relacionada ao fumo tenha duas etapas: a inicial, que aconteceria no epitélio e na submucosa, com participação de neutrófilos e de macrófagos; e a tardia, com a participação adicional dos linfócitos. ${ }^{(16,17)}$ Nossos achados demonstram que os linfócitos T CD8 ${ }^{+}$e os neutrófilos estão significativamente aumentados no escarro dos pacientes com DPOC, enquanto os tabagistas apresentam incremento de neutrófilos em relação ao grupo não-tabagista, o que também é citado na literatura. ${ }^{(18)}$ Desta forma, a presença aumentada do percentual de linfócitos T CD8 ${ }^{+}$e de neutrófilos no El poderia ajudar na identificação dos tabagistas que já possuem um modelo inflamatório semelhante ao que é observado em pacientes com DPOC. Na literatura, existe relato do aumento de neutrófilos no lavado broncoalveolar e no El de pacientes com DPOC, mas estes achados não são reproduzidos nos estudos que utilizam o material de biópsia brônquica. A principal justificativa para este fato refere-se à rápida migração dos neutrófilos do vaso para as vias aéreas, tornando-se mais fácil encontrá-los no interior das vias respiratórias do que na parede brônquica ou no parênquima pulmonar.

A técnica do $\mathrm{El}$ ainda não está padronizada para o estudo da inflamação em pacientes com DPOC, o que permite que os trabalhos realizados apresentem algumas diferenças com relação ao método empregado, tanto no processamento do material colhido como na quantificação das células. Além disso, existe a possibilidade de a solução salina hipertônica ser capaz de elevar o número de neutrófilos. Talvez o material mais adequado para a análise celular fosse o primeiro escarro da manhã, que representa o acúmulo de células, debris celulares e citocinas de grandes e pequenas vias respiratórias. ${ }^{(19,20)}$

A correlação entre o percentual de neutrófilos recolhido no $\mathrm{El}$ com o $\mathrm{VEF}_{1}$ foi inversamente propor- cional e fraca $\left(r^{2}=-0,108\right.$ e $\left.p=0,002\right)$. Este dado e as demais correlações celulares do El e do sangue venoso com os principais parâmetros espirométricos $\left(\mathrm{VEF}_{1}\right.$, CVF e VEF $/$ CVF) podem não refletir adequadamente o modelo inflamatório da DPOC, o qual predomina nas pequenas vias respiratórias. Como sabemos, esses parâmetros espirométricos não traduzem somente os distúrbios das pequenas vias. ${ }^{(11)}$ Além disso, o El avalia predominantemente as grandes vias aéreas. Logo, a correlação do estudo celular pela técnica do El e a espirometria podem não ser a forma ideal de se estudar a inflamação na DPOC, já que a localização inicial e preferencial do processo inflamatório ocorre nas pequenas vias aéreas.

Neste estudo, a contagem absoluta e percentual de macrófagos foi menor entre os pacientes com DPOC. Como a população de macrófagos recolhida no El de indivíduos sem doença respiratória representa a quase totalidade das células, é compreensível que, em pacientes com DPOC, este percentual esteja diminuído, por conta do aumento das outras populações celulares. ${ }^{(13,21)}$

Os macrófagos alveolares dos pacientes com DPOC são pleomórficos e, em muitos casos, contêm pigmentos antracóticos no interior do seu citoplasma (vide Figura 1 seção A). Este achado constituiu-se no principal fator para a teoria antigênica macrofágica. ${ }^{(16,22,23)} 0$ s macrófagos se ativariam e recrutariam outras células para a proteção pulmonar, mas como estas partículas de antracose não são efetivamente depuradas, o processo inflamatório se perpetuaria. 0 que dificulta a aceitação consensual desta teoria é que nem todos os tabagistas desenvolvem a DPOC, nem é a doença diretamente relacionada à carga-tabágica.

0 nosso achado de células T CD8 ${ }^{+}$aumentadas no El e no sangue periférico apóia a teoria do envolvimento sistêmico da DPOC, já que a migração dessas células se faz do vaso para o tecido pulmonar, e o seu processo de diferenciação e proliferação ocorre principalmente em outros sítios, especialmente na medula óssea. ${ }^{(6,7,24)}$ Contudo, o reduzido número de linfócitos normalmente encontrado no El dificulta a sua valorização.

0 linfócito $\mathrm{T} \mathrm{CD}^{+}$apresenta várias funções. $\mathrm{Na}$ DPOC, a teoria ainda mais prevalente refere-se a sua participação como célula de defesa (citotóxica) nas freqüentes infecções virais. ${ }^{(6)}$ No entanto, não se sabe o porquê da sua permanência no pulmão. Os linfócitos T $\mathrm{CD}^{+}{ }^{+}$ativados conseguem aumentar 
a taxa de apoptose das células $\mathrm{T} \mathrm{CD}^{+}$, diminuindo a proporção $\mathrm{CD} 4 / \mathrm{CD} 8$. Este mecanismo é dependente da expressão de Fas e não é antígeno específico. (25) Talvez, a permanência dos linfócitos T $\mathrm{CD} 8^{+}$nos pacientes com DPOC se dê pelo mecanismo imune conhecido como tolerabilidade antigênica, isto é, se o estímulo antigênico é persistente e algumas vezes muito intenso, as células T CD8 ${ }^{+}$regulariam a intensidade da resposta imunológica.

Em pacientes com doença estável, alguns trabalhos têm demonstrado aumento de eosinófilos no escarro, no lavado broncoalveolar e na parede das vias aéreas. ${ }^{(13)}$ Há observações de que fumantes não-asmáticos podem ter maior sensibilização para certos alérgenos ocupacionais e apresentar valores de imunoglobulina $\mathrm{E}$ e de eosinófilos sangüíneos maiores do que os dos não-tabagistas e indivíduos sem antecedentes alérgicos. ${ }^{(26,27)}$ No entanto, outros autores não conseguiram reproduzir estes dados. $(13,28)$ Outros ainda ${ }^{(29)}$ relataram o aumento dessas células em alguns pacientes, sugerindo que a eosinofilia no escarro poderia estar relacionada a uma melhor resposta ao tratamento com corticóide. No presente estudo, observou-se tendência de aumento dessas células no material recolhido das vias respiratórias dos pacientes com obstrução brônquica $(3,97 \pm 8,35 \%)$, o que não pode ser atribuído à infecção pulmonar, já que os pacientes estavam há mais de 1 mês sem exacerbações. Assim, os eosinófilos podem participar do processo inflamatório na DPOC também nos períodos de estabilidade clínica. ${ }^{(29,30)} \mathrm{A}$ análise do sangue periférico demonstrou aumento significativo dos eosinófilos ( $p=0,021)$, sendo que os valores leucométricos percentuais $\mathrm{e}$ totais se encontravam dentro da faixa da normalidade, fato que também fortalece a caracterização da participação eosinofílica na DPOC.

Em conclusão, os neutrófilos, eosinófilos, macrófagos e linfócitos $\mathrm{T} \mathrm{CD}^{+}$se encontravam aumentados no El, o que aponta para um modelo inflamatório de envolvimento multicelular na patogenia da DPOC. As células T CD8 ${ }^{+}$também estavam aumentadas no sangue periférico no período de estabilidade da doença. Este dado sugere que esta célula possa servir como marcador da inflamação sistêmica e ajudar na identificação precoce dos tabagistas, que já apresentam padrão inflamatório de DPOC. Para isto, novos estudos são necessários para a melhor caracterização do papel dos linfócitos na patogenia da DPOC.

\section{Agradecimentos}

Aos médicos Alberto José de Araújo e Arnaldo José Noronha Filho, ao setor de Prova de Função Respiratória do HUCFF/UFRJ e à equipe do Laboratório Multidisciplinar de Pesquisa da UFRJ: Patrícia Martins Lago, Vera Cristina da Silva Flores Batista, Lorena de Oliveira Rego Peçanha, Cesônia de Assis Martinusso e Alessandra Ferreira Coelho.

\section{Referências}

1. GOLD - Global strategy for the diagnosis, management, and prevention of chronic obstructive pulmonary disease [Homepage on the Internet]. Executive Summary, Global Strategy for the Diagnosis, Management, and Prevention of COPD Updated 2005 [cited 2006 Sep 9]. Available from: http://www.goldcopd.org/Guidelineitem. asp? $11=2 \& t 12=1$ \& tintld $=1662$

2. Walker Rl, Willemze R. Neutrophil kinetics and the regulation of granulopoiesis. Rev Infect Dis. 1980;2(2):282-92.

3. Cosio MG, Guerassimov A. Chronic obstructive pulmonary disease. Inflammation of small airways and lung parenchyma. Am J Respir Crit Care Med. 1999;160(5 Pt 2):S21-5.

4. Cosio M, Ghezzo H, Hogg JC, Corbin R, Loveland M, Dosman $\mathrm{J}$, et al. The relations between structural changes in small airways and pulmonary-function tests. $\mathrm{N}$ Engl J Med. 1978;298(23):1277-81.

5. Janoff A. Elastases and emphysema. Current assessment of the protease-antiprotease hypothesis. Am Rev Respir Dis. 1985;132(2):417-33.

6. Barnes PJ, Shapiro SD, Pauwels RA. Chronic obstructive pulmonary disease: molecular and cellular mechanisms. Eur Respir J. 2003;22(4):672-88.

7. Hogg JC. Pathophysiology of airflow limitation in chronic obstructive pulmonary disease. Lancet. 2004;364(9435):709-21.

8. Shapiro SD. Evolving concepts in the pathogenesis of chronic obstructive pulmonary disease. Clin Chest Med. 2000;21(4):621-32.

9. Di Stefano A, Capelli A, Lusuardi M, Balbo P, Vecchio C, Maestrelli $\mathrm{P}$, et al. Severity of airflow limitation is associated with severity of airway inflammation in smokers. Am J Respir Crit Care Med. 1998;158(4):1277-85.

10. Turato G, Zuin R, Miniati M, Baraldo S, Rea F, Beghe B, et al. Airway inflammation in severe chronic obstructive pulmonary disease: relationship with lung function and radiologic emphysema. Am J Respir Crit Care Med. 2002;166(1):105-10.

11. Amadori A, Zamarchi R, De Silvestro G, Forza G, Cavatton G, Danieli GA, et al. Genetic control of the CD4/CD8 T-cell ratio in humans. Nat Med. 1995;1(12):1279-83.

12. Pin 1, Gibson PG, Kolendowicz R, Girgis-Gabardo A, Denburg JA, Hargreave FE, et al. Use of induced sputum cell counts to investigate airway inflammation in asthma. Thorax. 1992;47(1):25-9.

13. O’Donnell R, Breen D, Wilson S, Djukanovic R. Inflammatory cells in the airways in COPD. Thorax. 2006;61(5):448-54. 
14. Lung function testing: selection of reference values and interpretative strategies. American Thoracic Society. Am Rev Respir Dis. 1991;144(5):1202-18.

15. Hogg JC, Chu F, Utokaparch S, Woods R, Elliott WM, Buzatu L, et al. The nature of small-airway obstruction in chronic obstructive pulmonary disease. $\mathrm{N}$ Engl $\mathrm{J}$ Med. 2004;350(26):2645-53.

16. O'Shaughnessy TC, Ansari TW, Barnes NC, Jeffery PK. Inflammation in bronchial biopsies of subjects with chronic bronchitis: inverse relationship of CD8+ T lymphocytes with FEV . Am J Respir Crit Care Med. 1997;155(3):852-7.

17. Saetta M, Di Stefano A, Turato G, Facchini FM, Corbino L, Mapp CE, et al. CD8+ T-lymphocytes in peripheral airways of smokers with chronic obstructive pulmonary disease. Am J Respir Crit Care Med. 1998;157(3 Pt 1):822-6.

18. Bosken $\mathrm{CH}$, Hards J, Gatter K, Hogg JC. Characterization of the inflammatory reaction in the peripheral airways of cigarette smokers using immunocytochemistry. Am Rev Respir Dis. 1992;145(4 Pt 1):911-7.

19. Kips JC, Peleman RA, Pauwels RA. Methods of examining induced sputum: do differences matter? Eur Respir J. 1998;11(3):529-33.

20. Parameswaran K, Pizzichini MM, Li D, Pizzichini E, Jeffery PK, Hargreave FE. Serial sputum cell counts in the management of chronic airflow limitation. Eur Respir J. 1998;11(6):1405-8.

21. Barnes PJ. Mediators of chronic obstructive pulmonary disease. Pharmacol Rev. 2004;56(4):515-48.
22. Cosio MG, Majo J, Cosio MG. Inflammation of the airways and lung parenchyma in COPD: role of $\mathrm{T}$ cells. Chest. 2002;121(5 Suppl):160S-165S.

23. Hogg JC, Senior RM. Chronic obstructive pulmonary disease - part 2: pathology and biochemistry of emphysema. Thorax. 2002;57(9):830-4.

24. Shaw RJ, Djukanovic R, Tashkin DP, Millar AB, du Bois RM, Orr PA. The role of small airways in lung disease. Respir Med. 2002 Feb;96(2):67-80.

25. Liu CC, Young LH, Young JD. Lymphocyte-mediated cytolysis and disease. N Engl J Med. 1996;335(22):1651-9.

26. O'Connor GT, Sparrow D, Weiss ST. The role of allergy and nonspecific airway hyperresponsiveness in the pathogenesis of chronic obstructive pulmonary disease. Am Rev Respir Dis. 1989;140(1):225-52.

27. Sluiter HJ, Koëter GH, de Monchy JG, Postma DS, de Vries K, Orie NG. The Dutch hypothesis (chronic non-specific lung disease) revisited. Eur Respir J. 1991;4(4):479-89.

28. Saetta M. Airway inflammation in chronic obstructive pulmonary disease. Am J Respir Crit Care Med. 1999;160(5 Pt 2):S17-20.

29. Pizzichini E, Pizzichini MM, Gibson P, Parameswaran K, Gleich GJ, Berman L, et al. Sputum eosinophilia predicts benefit from prednisone in smokers with chronic obstructive bronchitis. Am J Respir Crit Care Med. 1998;158(5 Pt 1):1511-7.

30. Saetta M, Di Stefano A, Maestrelli P, Turato G, Ruggieri MP, Roggeri A, et al. Airway eosinophilia in chronic bronchitis during exacerbations. Am J Respir Crit Care Med. 1994;150(6 Pt 1):1646-52. 